hep-ph/0103181

\title{
Pion Production by Parametric Resonance Mechanism with Quantum Back Reactions
}

\author{
Hideaki Hiro-Oka円 \\ Institute of Physics, Kitasato University \\ 1-15-1 Kitasato Sagamihara, Kanagawa 228-8555, Japan \\ Hisakazu Minakataf \\ Department of Physics, Tokyo Metropolitan University \\ 1-1 Minami-Osawa, Hachioji, Tokyo 192-0397, Japan \\ and \\ Research Center for Cosmic Neutrinos, Institute for Cosmic Ray Research, \\ University of Tokyo, Kashiwa, Chiba 277-8582, Japan
}

\begin{abstract}
We investigate the problem of quantum back reactions due to particle production by the parametric resonance mechanism in an environment of nonequilibrium chiral phase transition. We work with the linear sigma model and employ Boyanovsky et al.'s formalism to take account of back reactions under the Hartree-Fock approximation. We calculate the single pion momentum distributions and two pion correlations under the initial condition of thermal equilibrium and small amplitude sigma oscillations around a potential minimum. We observe that the resonance peak survives under the back reaction which is remarkable considering the strong coupling of the sigma model.
\end{abstract}

\subsection{Ef,25.75.Gz,25.75.Dw}

\footnotetext{
${ }^{1}$ hiro-oka@phys.clas.kitasato-u.ac.jp

${ }^{2}$ minakata@phys.metro-u.ac.jp
} 


\section{Introduction}

It has been suggested that occurrence of nonequilibrium chiral phase transition in hadronic collisions would lead to the formation of disoriented chiral condensate (DCC), domains of coherent pion fields [1]. It then may offer a viable explanation of the Centauro events found in cosmic ray experiments which have anomalously large fluctuations of neutral to charged pion ratio [2].

By a numerical simulation using the linear sigma model, Rajagopal and Wilczek [3] demonstrated that low-momentum components of pion fields are greatly amplified, indicating the formation of large coherent domains of pions. A key ingredient in their simulation is the "quench" initial condition which models a hypothesized rapid cooling of hot debris formed during the hadronic reactions. The formation of large domains of pion fields was confirmed by more realistic but still the sigma model simulation by Asakawa, Huang, and Wang [4] in which the longitudinal expansion of hadronic blob is taken into account.

There remain, however, questions regarding the interpretation of the simulations. Among other things, the amplification of the low-momentum modes in the Rajagopal-Wilczek simulation lasts so long, much longer than $1 / m_{\sigma}$, the time scale of rolling down from the top of the Mexican hat potential. In previous works [5, 6], we have conjectured that the parametric resonance mechanism might be relevant for the DCC formation. In simple physical terms, the parametric resonance mechanism stands for a mechanism of amplification of pion field fluctuations due to cooperative effects coupled with oscillations of background sigma model fields.

It has been known since long time ago that the parametric resonance mechanism can be relevant for cosmology by providing reheating mechanism during inflation [7]. It has been received renewed interests recently in relationship with various issues, such as the gravitino problem, in inflationary cosmology [8]-10]. The parametric resonance mechanism for pion production was first discussed by Mrówczyński and Müller in close analogy to the out-of-equilibrium phase transition in cosmology [11]. We have stressed in our previous paper [5] that it may offer an efficient mechanism of pion production with potential possibility of explaining long-lasting amplification of low momentum modes. We also noted that it would give an alternative picture of large isospin fluctuations, the picture quite different from the conventional one based on rolling down into the "wrong" direction in isospin space and the subsequent relaxation to the true vacuum. We have discussed that the discriminative signature can be provided by the two pion correlations in back-to-back momentum configurations [5, 12]. 
In this paper, we investigate the effect of quantum back reactions onto the parametric resonance mechanism. Particle creation, when it occurs due to strong coupling with background field oscillations, affects the motion of the background fields by acting as dissipation. The back reaction, in turn, affects the particle production itself because of the damping of background oscillations. We aim at taking account of the interplay between particle production, its back reactions to the background fields, and reaction back to the particle production in a self consistent manner. Unfortunately, the effect was completely ignored in our previous treatment, but evaluation of its effects is indispensable for the correct understanding of a role played by the parametric resonance mechanism on DCC formation. This is our first trial toward the goal and we explore in this paper the effect of back reactions on single particle momentum distributions of pions, with primary concern on its effect on resonance peaks. We will also calculate correlation length of pions, one of the key issues in DCC.

In our previous works, our formulation relied on the approximation of small background sigma oscillations. It may correspond to the final stage of nonequilibrium chiral phase transition and we discussed the quantum particle creation under the background. We have argued that in this setting we may ignore higher order terms in the quantum pion and sigma fluctuations. Due to a series of approximations, we are able to write down explicit quantum states of pions and sigmas in the form of the squeezed states [13. It enables us to compute explicitly two pion correlations, and the BCS type back-to-back momentum pairing is obtained. It would give a characteristic signature of the parametric resonance mechanism.

In this paper, we calculate the effect of quantum back reactions but still within the framework of small amplitude background sigma oscillations. Our restriction to the small-amplitude region is not only due to technical reasons, but also is physically motivated. To understand the effects of quantum back reactions, it is desirable to compare the results with and without back reactions at the same initial amplitude of background $\sigma$ oscillation. Of course, the limitation clearly implies that the meaning of our results must be carefully interpreted, in particular with regard to possible implications to experiments.

We use the formulation by Boyanovsky et al. [9] which incorporates the quantum back reactions via the Hartree-Fock approximation. In our previous computation ignoring back reactions, the pion momentum distributions have resonance peaks at the momenta which are characteristic to the parametric resonance mechanism. If observed, it would give an unambiguous confirmation 
of the mechanism. However, it is possible that it can be wiped out when the effect of quantum back reaction is taken into account. For related works which address DCC by the similar formalism, see e.g., Refs. [14, 15, 16].

We will observe that the quantum back reactions drastically affect low momentum components of the pion field fluctuations but not on the resonance peaks. The peak hight is barely changed but the peak position is moved to lower momentum. We believe that the fact that the resonance peak survives even if the quantum back reactions are taken into account gives us some positive hints about experimental detectability of the parametric resonance mechanism.

Our calculation of two pion correlations results in a short correlation length of order 1-2 fm which is much shorter compared to the one expected in DCC scenario. We will briefly address its possible interpretation, in particular in the relationship with the different result obtained by Kaiser [14].

In section 2, we review the Boyanovsky et al.'s formalism. In section 3, we calculate the single pion momentum distributions and two pion correlations. In the last section, we give concluding remarks.

\section{Quantum Back Reactions in the Hartree- Fock Approximation}

We start with the following linear sigma model Lagrangian

$$
\mathcal{L}=\frac{1}{2} \partial_{\mu} \phi_{a} \partial^{\mu} \phi_{a}-V(\phi),
$$

where

$$
V(\phi)=\frac{\lambda}{4}\left(\phi_{a} \phi_{a}-v_{0}^{2}\right)^{2}+h \sigma, \quad \phi_{a}=(\sigma, \vec{\pi}) .
$$

Typical values of the parameters

$$
\lambda=20, \quad v_{0}=90 \mathrm{MeV}, \quad m_{\pi}=\sqrt{\frac{h}{v_{0}}}=140 \mathrm{MeV},
$$

are employed throughout this paper, the same ones as used in our previous analysis. [5]. We rely on the formalism based on the Schrödinger picture formulation of quantum field theory [9].

In the following calculations, we consider the pion production in a background sigma field which is oscillating along the sigma direction in isospin 
space. Decomposing sigma model fields into the background and fluctuations, $\sigma=\varphi_{0}+\varphi$ and $\vec{\pi}=\vec{\pi}$, we substitute them into the Lagrangian (1). We employ the Hartree-Fock approximation to take account of the back reaction. Then, the quartic terms in the Lagrangian can be replaced by quadratic terms, e.g., $\varphi^{4} \rightarrow 6\left\langle\varphi^{2}\right\rangle \varphi^{2}-3\left\langle\varphi^{2}\right\rangle^{2}$. The Hamiltonian is expressed as

$$
\begin{aligned}
\mathcal{H}= & \frac{1}{2} \Pi_{\varphi}^{2}+\frac{1}{2} \Pi_{\pi}^{2}+\frac{1}{2}(\nabla \varphi)^{2}+\frac{1}{2}(\nabla \pi)^{2}+\frac{1}{2} \mathcal{M}_{\varphi}^{2}(t) \varphi^{2}+\frac{1}{2} \mathcal{M}_{\pi}^{2}(t) \pi^{2} \\
& +\left(\lambda\left(3\left\langle\varphi^{2}\right\rangle+\left\langle\pi^{2}\right\rangle\right) \varphi_{0}+\left.\frac{\partial V(\sigma, 0)}{\partial \sigma}\right|_{\sigma=\varphi_{0}}\right) \varphi-\frac{3}{4} \lambda\left(\left\langle\varphi^{2}\right\rangle^{2}+\left\langle\pi^{2}\right\rangle^{2}\right)+V\left(\varphi_{0}, 0\right)
\end{aligned}
$$

where ח's stand for the conjugate momenta of fields indicated as indices. The time dependent effective masses for sigma and pion are written, respectively, as

$$
\begin{aligned}
& \mathcal{M}_{\sigma}^{2}=\lambda\left(3 \varphi_{0}^{2}-v_{0}^{2}\right)+3 \lambda\left\langle\varphi^{2}\right\rangle, \\
& \mathcal{M}_{\pi}^{2}=\lambda\left(\varphi_{0}^{2}-v_{0}^{2}\right)+3 \lambda\left\langle\pi^{2}\right\rangle .
\end{aligned}
$$

The expectation value is evaluated by

$$
\left\langle\varphi^{2}\right\rangle=\operatorname{Tr}\left(\varphi^{2} \rho\right)
$$

where $\rho$ is a functional density matrix written in terms of $\varphi$. In a self-consistent Hartree approximation, the density matrix in sigma sector takes the Gaussian form 9

$\rho_{\sigma}[\sigma, \tilde{\sigma}]=\prod_{k} \mathcal{N}_{\varphi} \exp \left[-\frac{\mathcal{A}_{\varphi}}{2} \varphi_{k} \varphi_{-k}-\frac{\mathcal{A}_{\varphi}^{*}}{2} \tilde{\varphi}_{k} \tilde{\varphi}_{-k}-\mathcal{B}_{\varphi} \varphi_{k} \tilde{\varphi}_{-k}+i \Pi_{\varphi k}\left(\varphi_{-k}-\tilde{\varphi}_{-k}\right)\right]$,

where $\mathcal{N}_{\varphi}, \mathcal{A}_{\varphi}, \mathcal{A}_{\varphi}^{*}$, and $\mathcal{B}_{\varphi}$ are determined so that the density matrix obeys the functional Liouville equation

$$
i \frac{\partial \rho}{\partial t}=[\mathcal{H}, \rho] .
$$

For instance, we have

$$
\begin{gathered}
i \dot{\mathcal{N}}_{\varphi}=\frac{1}{2} \mathcal{N}_{\varphi}\left(\mathcal{A}_{\varphi}-\mathcal{A}_{\varphi}^{*}\right), \\
-\dot{\Pi}_{\varphi k}=\left(\left(3 \lambda\left\langle\varphi^{2}\right\rangle+3 \lambda\left\langle\pi^{2}\right\rangle\right) \varphi_{0}+\left.\frac{\partial V(\sigma, 0)}{\partial \sigma}\right|_{\sigma=\varphi_{0}}\right) \sqrt{\Omega} \delta_{k 0},
\end{gathered}
$$


where $\Omega$ is a finite volume of the system. Actually, the equation (10) leads to the following equation of motion of $\varphi_{0}$

$$
\ddot{\varphi}_{0}+\lambda\left(\varphi_{0}^{2}-v_{0}^{2}\right) \varphi_{0}+\left(3 \lambda\left\langle\varphi^{2}\right\rangle+3 \lambda\left\langle\vec{\pi}^{2}\right\rangle\right) \varphi_{0}-h=0 .
$$

As was done in the analysis by Boyanovsky et al. [9], the assumption of no cross correlations between sigma and pion fields is adopted in the derivation of the above effective Hamiltonian (4). Thanks to this assumption, the density matrices of sigma and pion sectors are factorized, allowing their separate treatment. Yet, the interaction between sigma and pion fields influences the dynamics through motion of $\varphi_{0}$, whose behavior is strongly affected by quantum back reactions due to particle productions. Among other things, it ensures the conservation of total energy despite the factorization of sigma and pion sectors. To briefly summarize, the effects of higher order terms of fields are included under the Hartree-Fock approximation, and the back reaction due to particle production is taken into account through the mean field values evaluated with use of the density matrix.

The expectation value of sigma fluctuation is expressed as

$$
\begin{aligned}
\left\langle\varphi^{2}\right\rangle & =\frac{1}{\Omega} \sum_{k}\left\langle\varphi_{k} \varphi_{-k}\right\rangle \\
& =\frac{1}{\Omega} \sum_{k} \operatorname{Tr}\left(\varphi_{k} \varphi_{-k} \rho_{\sigma}\right) .
\end{aligned}
$$

After a short calculation, which is straightforward with the Gaussian density matrix, we find

$$
\left\langle\varphi_{k} \varphi_{-k}\right\rangle=\frac{1}{2}\left|\psi_{\varphi}\right|^{2} \operatorname{coth}\left(\frac{\beta_{0} \omega_{\varphi}(k, 0)}{2}\right)
$$

under the assumption that the initial state is in thermal equilibrium with a temperature $T_{0}=1 / \beta_{0}$. This assumption can be expressed in terms of coefficients of the density matrix as follows:

$$
\begin{aligned}
\mathcal{A}_{\varphi}(k, 0) & =\mathcal{A}_{\varphi}^{*}(k, 0)=\omega_{\varphi}(k, 0) \operatorname{coth} \beta_{0} \omega_{\varphi}(k, 0) \\
\mathcal{B}_{\varphi}(k, 0) & =-\omega_{\varphi}(k, 0) \operatorname{cosech} \beta_{0} \omega_{\varphi}(k, 0) \\
\mathcal{N}_{\varphi}(k, 0) & =\left(\frac{\omega_{\varphi}(k, 0)}{\pi} \tanh \frac{\beta_{0} \omega_{\varphi}(k, 0)}{2}\right)^{1 / 2}
\end{aligned}
$$

The function $\psi_{\varphi}$ defined by

$$
-i \frac{\dot{\psi}_{\varphi}}{\psi_{\varphi}}=\operatorname{Re} \mathcal{A}_{\varphi} \cdot \tanh \beta_{0} \omega_{\varphi}(k, 0)+i \operatorname{Im} \mathcal{A}_{\varphi}
$$


obeys the equation of motion of $\varphi$,

$$
\ddot{\psi}_{\varphi}(k, t)+\omega_{\varphi}^{2}(k, t) \psi_{\varphi}(k, t)=0,
$$

with

$$
\omega_{\varphi}^{2}(k, t)=k^{2}+\mathcal{M}_{\varphi}^{2}(t) .
$$

The initial condition for $\psi_{\varphi}$ is given by

$$
\psi_{\varphi}(k, 0)=\frac{1}{\sqrt{\omega_{\varphi}(k, 0)}}, \quad \dot{\psi}_{\varphi}(k, 0)=i \sqrt{\omega_{\varphi}(k, 0)},
$$

through the initial conditions for the density matrix under the assumption of thermal equilibrium. The point is that the function $\psi$ satisfies the classical equation thanks to the transformation (17), despite that it describes the quantum system. As one can see, the right hand side of the equation (13) contains the expectation value of $\varphi^{2}$ at $t=0$ through the effective mass dependence in the frequency (19). Hence, the initial value of $\left\langle\varphi^{2}\right\rangle$ must be determined self-consistently.

Let us consider an expectation value of the pion number $\left\langle n_{\vec{\pi}}(k, t)\right\rangle$. A particle picture in a time dependent background, which corresponds to the time-dependent mass (5), has the similar feature as those in theories in curved space-time. The effective mass has the similar form as the coefficient of the quadratic term of scalar fields coupled with the Ricci scalar [8]. In this case, the Fock spaces chosen in both of the asymptotic regions $(t \rightarrow \pm \infty)$ belong to different Hilbert spaces. A vacuum in one of the Fock spaces is a condensed state of particles defined in the other Fock vacuum. The creation and annihilation operators in each Fock space are related via the Bogoliubov transformation. Two different definitions of this transformation are employed in several literatures so far [9]-[16].

In general, the Bogoliubov transformation between operators in two Fock spaces is written as

$$
a_{k}(t)=\alpha_{k}(t) a_{0 k}+\beta_{k}^{*}(t) a_{0-k}^{\dagger}, \quad a_{k}^{\dagger}(t)=\beta_{k}(t) a_{0-k}+\alpha_{k}^{*}(t) a_{0 k}^{\dagger},
$$

where each of $a_{k}$ and $a_{0 k}$ belongs to a different Fock space and the coefficients obey $\left|\alpha_{k}(t)\right|^{2}-\left|\beta_{k}(t)\right|^{2}=1$. From the transformation (21), the number operator $a_{k}^{\dagger}(t) a_{k}(t)$ is of the form

$$
a_{k}^{\dagger}(t) a_{k}(t)=\left(\left|\alpha_{k}(t)\right|^{2}+\left|\beta_{k}(t)\right|^{2}\right) a_{k}^{\dagger} a_{k}+\left|\beta_{k}(t)\right|^{2} .
$$


A possible choice of coefficients of the Bogoliubov transformation is given by

$$
\begin{aligned}
& \alpha_{k}(t)=\frac{1}{2 \sqrt{\omega_{\vec{\pi}}(k, 0)}}\left(i \dot{\psi}_{\vec{\pi}}^{*}+\omega_{\vec{\pi}}(k, 0) \psi_{\vec{\pi}}^{*}\right), \\
& \beta_{k}(t)=\frac{1}{2 \sqrt{\omega_{\vec{\pi}}(k, 0)}}\left(i \dot{\psi}_{\vec{\pi}}+\omega_{\vec{\pi}}(k, 0) \psi_{\vec{\pi}}\right),
\end{aligned}
$$

and the other possibility is as follows

$$
\begin{aligned}
& \alpha_{k}(t)=\frac{1}{2 \sqrt{\omega_{\vec{\pi}}(k, t)}}\left(i \dot{\psi}_{\vec{\pi}}^{*}+\omega_{\vec{\pi}}(k, t) \psi_{\vec{\pi}}^{*}\right) e^{i \int^{t} d t^{\prime} \omega_{\vec{\pi}}\left(k, t^{\prime}\right)}, \\
& \beta_{k}(t)=\frac{1}{2 \sqrt{\omega_{\vec{\pi}}(k, t)}}\left(i \dot{\psi}_{\vec{\pi}}+\omega_{\vec{\pi}}(k, t) \psi_{\vec{\pi}}\right) e^{-i \int^{t} d t^{\prime} \omega_{\vec{\pi}}\left(k, t^{\prime}\right)} .
\end{aligned}
$$

The former definition (23) relies on time evolutions of canonical fields, e.g., $\vec{\pi}(k, t)=U(t) \vec{\pi}(k, 0) U^{-1}(t)$, and Boyanovsky et al. utilizes this type of the number operator for the numerical analysis. Here $U(t)$ is a time evolution operator. In the latter definition, the creation and annihilation operators diagonalize the Hamiltonian at any time $t$ [17], and $a^{\dagger} a$ defined by these coefficients is often referred to as adiabatic number operator. We will choose the second option, the transformation coefficients (24) as we did in our previous papers. By definition, both number operators coincide at $t=0$.

A subtle problem, however, arises in the latter definition. As noticed in the previous papers [12, 5], there exists a possibility that $\omega(k, t)$ becomes imaginary when the amplitude of the background sigma oscillation exceeds a limit, $\left(1-\varphi_{0} / v\right)^{2}>\left(m_{\pi} / m_{\sigma}\right)^{2}$. It is highly plausible that the ill-defined imaginary frequency is an artifact of the wrong choice of variables, but we do not enter into the problem in this paper.

\section{Single Pion Momentum Distributions and Two Pion Correlations}

The expectation value of the pion number operator is defined by

$$
\left\langle n_{\vec{\pi}}(k, t)\right\rangle=\operatorname{Tr}\left(a_{k}^{\dagger}(t) a_{k}(t) \rho(0)\right),
$$

where $\rho(0)$ stands for the functional density matrix at $t=0$. At $t=0$, the initial occupation number is given by

$$
\left\langle n_{\vec{\pi}}(k, 0)\right\rangle=\frac{1}{e^{\beta_{0} \omega_{\vec{\pi}}(k, 0)}-1},
$$


at finite temperature via the first term of right-hand-side in (22), an expected result. It is easy to obtain the energy in pion sector in terms of a function $\psi_{\vec{\pi}}$, which is defined similarly as $\psi_{\varphi}$ in (17), and it is given by

$$
E_{\vec{\pi}}(k, t)=\left(\frac{1}{2}\left|\dot{\psi}_{\vec{\pi}}(k, t)\right|^{2}+\frac{1}{2} \omega_{\vec{\pi}}^{2}(k, t)\left|\psi_{\vec{\pi}}(k, t)\right|^{2}\right) /\left(e^{\beta_{0} \omega_{\vec{\pi}}(k, 0)}-1\right) .
$$

We now proceed to the numerical analysis. We restrict ourselves into small initial background oscillation of the sigma field. We parametrize it, throughout the remaining sections, by the parameters

$$
\frac{\chi_{0}(0)}{v} \equiv \frac{\varphi_{0}-v}{v}
$$

which implies a departure from the bottom of the potential minimum measured in units of $v$, the vacuum expectation value of $\varphi$. By solving the coupled differential equations (11) and (18), we compute $\left\langle n_{\vec{\pi}}(k, t)\right\rangle$. In Fig. 1, we present snapshots of the single pion momentum distributions at time $t=0$, $2,4,6,8$, and $10 \mathrm{fm}$ with the initial amplitude of a background oscillation $\chi_{0}(0) / v=0.05$. This is the same initial condition as in the previous analysis ignoring the back reaction [5] and the snapshots obtained there at $t=4,7$, and $10 \mathrm{fm}$ are presented in Fig. 2 for comparison. (At $t=0,\left\langle n_{\vec{\pi}}(k, t)\right\rangle$ trivially vanishes because of the initial condition chosen in [5].) From Fig. 1, one observes the three significant characteristics of the time evolution of the pion momentum distribution:

(1) At momenta lower than $\sim 100 \mathrm{MeV}$, the number density grows first and then decreases, and seems to undergo damped oscillations. The suppression of number density at low momenta seems to indicate that the back reaction due to particle creations are efficient at low momenta. It may be a bad news for DCC because it may suppress the formation of large coherent domains. We will make some comments later.

(2) At around the resonance energy $\sim 200 \mathrm{MeV}$, a peak starts to develop at $t \simeq 4 \mathrm{fm}$ and continues to grow as time goes by. The series of snapshots in Fig. 1 looks like a process that the peak grows by absorbing the ambient pions in low momentum region which was originally provided, as seen by comparing with Fig. 2, by the background oscillation in an early stage of evolution.

(3) At momenta larger than $\sim 300 \mathrm{MeV}$, there is virtually no change in the initial thermal distribution. 
The peak height of pion number distribution at resonance without the back reaction reaches 0.28 at $t=10 \mathrm{fm}$ in Fig. 2. The corresponding peak height with back reaction is 0.38 at $t=10 \mathrm{fm}$ as shown in Fig. 1 . The peak height with the back reaction is higher but it is mainly due to the fact that the initial occupation number (26) is nonzero. If we estimate the net increase of height due to evolution just by subtracting the initial thermal distribution, we obtain 0.25 . It amounts to about $90 \%$ of the peak height without the back reaction.

We also note, by comparing Fig. 1 with Fig. 2, that the peak position moves to lower momentum region when the back reaction is taken into account. It can be understood by the following consideration.

If we ignore back reactions, the equation of motion of pion fields reduces to the Mathieu equation

$$
\left(\frac{d^{2}}{d z^{2}}+A-2 q \cos (2 z)\right) \pi_{k}=0,
$$

where $A=4\left(k^{2}+m_{\pi}^{2}\right) / m_{\sigma}^{2}$ and $q=4 \lambda v \chi_{0}(0) / m_{\sigma}^{2}$ [5]. Here we used a dimensionless time variable defined by $z=m_{\sigma} t / 2$. It is well known that the equation admits unstable solutions in a wide range of parameters, in particular at $A=n^{2}$ for small $q$ which is relevant to our case, where $n$ denotes an arbitrary integer [18]. Using the effective pion mass corrected by back reactions (5) the peak position is roughly estimated as

$$
k_{\text {peak }} \sim \sqrt{\frac{m_{\sigma}^{2}}{4}-m_{\pi}^{2} \cdot \frac{v_{0}}{v}-\lambda \chi_{0}^{2}-3 \lambda\left\langle\pi^{2}\right\rangle},
$$

for the first resonance band $A=1$.

The back reaction affects $k_{\text {peak }}$ in two opposite ways. The amplitude of the oscillation obviously damps by back reactions and it makes the third term in the square root smaller in magnitude at $t \neq 0$, and hence $k_{\text {peak }}$ larger. On the other hand, the last term, which was absent in a case of no back reaction, tends to let $k_{\text {peak }}$ be smaller. A simple estimate shows that the latter effect wins.

To confirm this interpretation, we run the computations with four different initial conditions in the region $\chi_{0} / v \in[0.04,0.10]$. In Fig. 3, we plot the pion distributions at $t=20 \mathrm{fm}$. One notices that the peak position moves toward a lower momentum region by increasing the amplitudes of background sigma oscillation in consistent with our interpretation. It is also notable that the 
peak height rapidly increases as $\chi_{0} / v$ gets larger. It clearly shows a hint for experiments.

The behavior of damping of the background oscillation is shown in Fig. 4. This and the resultant increase of the effective mass cause a shift of the peak position to a lower momentum, as we just argued. In Fig. 5, we plot the time evolution of the energy of pion sector, $E_{\pi}$. It is defined as the summation over $k$ in (27). It increases while the background sigma oscillation damps with characteristic time scale of $\sim 10 \mathrm{fm}$, and becomes stationary at about $t \sim 15$ fm when the background sigma oscillations die away. The pion production effectively terminates at this point.

The momentum distribution for sigma fluctuations is shown in Fig. 6. It represents a sharp contrast with the behavior of pion distributions, having no evolution until $t=30 \mathrm{fm}$. The first resonance is expected at zero momentum, but it is not visible. It may be due to cancellation between the resonance enhancement and the damping due to back reactions at low momenta.

We have observed that the time scale of the energy dissipation is $\sim 10 \mathrm{fm}$. The time scale can roughly be understood by the following arguments. For simplicity, we consider a system with pion and background fields only. Suppose that the background fields oscillate harmonically. Using the virial theorem, the energy conservation reads

$$
m_{\sigma}^{2} \tilde{\chi}_{0}(0)^{2}+\epsilon_{\pi}(0)=m_{\sigma}^{2} \tilde{\chi}_{0}(z)^{2}+\epsilon_{\pi}(0) e^{2 \mu z}
$$

since the pion field $\pi_{k}(z)$ is expressed by using the critical exponent as $\pi_{k}(0) e^{\mu z}$ in a resonance band. The pion energy density $\epsilon_{\pi}$ is proportional to the square of the pion field $\pi_{k}(z)$. $\tilde{\chi}_{0}$ implies the amplitude of background oscillation, and $\mu$ is the critical exponent of the Mathieu function [18]. As mentioned above, the equation of motion of pion is described by the Mathieu equation in this simple system and the energy of pion is dominated by the modes in the resonance band in a good approximation.

In our analysis, a parametric resonance occurs in a narrow resonance band and $\mu$ is given by $\mu=q / 2$ [18], that is

$$
\mu=\frac{2 \lambda v \tilde{\chi}}{m_{\sigma}^{2}}=\frac{2 \lambda v \sqrt{\epsilon_{0}-\epsilon_{\pi}(z)}}{m_{\sigma}^{3}},
$$

where $\epsilon_{0}$ is the initial total energy density of the system considered. Because the time dependence of pion energy at any time $z$ is expressed by $\epsilon_{\pi}(z)=$ 
$\epsilon_{\pi}(0) e^{2 \mu z}$, one obtain the following equation

$$
\frac{d \epsilon_{\pi}(z)}{d z}=2 \mu \epsilon_{\pi}(z)
$$

in the region where the time derivative of $\mu$ can be neglected. Practically, it means the narrow resonance region. The solution is given by

$$
\epsilon_{\pi}(z)=\epsilon_{0}\left(1-\tanh ^{2}\left[-\frac{2 \lambda v \sqrt{\epsilon_{0}}}{m_{\sigma}^{3}} z+\tanh ^{-1} \sqrt{1-\frac{\epsilon_{\pi}(0)}{\epsilon_{0}}}\right]\right),
$$

where $z \in\left[0, z_{\text {end }}\right]$. The time $z_{\text {end }}$ is a dimensionless cut off time beyond which the equation (33) is no longer valid. One can see that almost all energy of background field is transferred to pions by the time given by

$$
z_{\text {end }}=\frac{m_{\sigma}^{3}}{2 \lambda v \sqrt{\epsilon_{0}}} \tanh ^{-1} \sqrt{1-\frac{\epsilon_{\pi}(0)}{\epsilon_{0}}} .
$$

The energy density $\epsilon_{0}$ is determined, once the initial amplitude of sigma oscillation is given. With parameters used in our numerical calculation, $\chi_{0} / v=0.05$, $\epsilon_{0} \simeq 8.67 \times 10^{6} \mathrm{MeV}^{4}$. Using the parameters given in (3), $t_{\text {end }}$ is estimated to be $\sim 8 \mathrm{fm}$. Note that this value is under-estimated because $\mu$ is treated as a constant in the method we used. Since $\mu$ decreases with time, the energy transfer needs longer time to be completed. Nevertheless, it is notable that the treatment gives a correct order of magnitude estimation of $z_{\text {end }}$.

Two point correlation function $C(t, r)$ is similarly obtained by means of the density matrix, i.e.,

$$
\begin{aligned}
C(t, r) & =\langle\vec{\pi}(t, 0) \vec{\pi}(t, r)\rangle \equiv \operatorname{Tr}(\vec{\pi}(t, 0) \vec{\pi}(t, r) \rho(0)) \\
& =\frac{1}{\Omega^{1 / 3}} \sum_{k} \frac{k^{2}}{2 \pi} j_{0}(k r)\left|\psi_{\pi}\right|^{2} \operatorname{coth}\left(\frac{\beta_{0} \omega_{\vec{\pi}}(k, 0)}{2}\right),
\end{aligned}
$$

where $j_{0}(x)=\sin x / x$ denotes the spherical Bessel function of order 0 . In Fig. 7 , we plot two point pion correlation functions at $t=5,10$, and $20 \mathrm{fm}$. At $t=10$ when the evolution effectively ends, the correlation length $\xi$ is given by $\xi \simeq 1.3 \mathrm{fm}$.

In search for the possibility of correlation length growth, we run the computations with varying initial conditions. The correlation length increases for larger initial background oscillations, but not so much, as we observe in Table.1. 
Table 1: Correlation length $\xi$ of pions at $t=20 \mathrm{fm}$ for varying initial background amplitude $\varphi_{0} / v$ 's.

\begin{tabular}{ccccccc}
\hline \hline$\chi_{0}(0) / v$ & 0.05 & 0.06 & 0.07 & 0.08 & 0.09 & 0.10 \\
$\xi[\mathrm{fm}]$ & 1.27 & 1.36 & 1.43 & 1.50 & 1.61 & 1.68 \\
\hline \hline
\end{tabular}

Apparently, this result is in disagreement with the conclusion reached by Kaiser who found the large domain formation due to the parametric resonance mechanism [14]. Our simulation indicates that the back reaction primarily affects low momentum components and it is unlikely that the mechanism itself leads to large domain formation.

We note, however, that there are two significant differences between our and his calculations:

(i) The initial amplitude of background sigma oscillation is large, $\chi_{0} / v \sim 1$ in Kaiser's calculations, whereas it is only up to 0.1 in our present analysis.

(ii) The quantum back reaction is fully taken into account in each step of evolution of the system under the Hartree-Fock approximation in our calculation. But it is ignored in Kaiser's analysis apart from that the effect is evaluated to determine the time at which the simulation has to stop.

At this stage, we feel it difficult to judge whether or not large coherent domains are expected to form by the parametric resonance mechanism. It may well be the case that it depends upon the initial conditions. To really address the issue, we have to run a simulation with large initial amplitudes with full taking account of quantum back reactions. To carry it out, we need a formulation which is free from the instability problem as we mentioned at the end of Sec. 2.

\section{Concluding Remarks}

In this paper we have investigated features of quantum back reactions due to particle production by the parametric resonance mechanism in an environment of nonequilibrium chiral phase transition. We have calculated the single pion momentum distributions and two pion correlations under the initial small am- 
plitude sigma oscillation around a potential minimum and with initial thermal equilibrium assumption. We relied on the formalism developed by Boyanovsky et al. to take account of back reactions under the Hartree-Fock approximation based on the Schrödinger picture formulation of quantum field theory. Remarkably, we observed that the resonance peak survives under the back reaction even in such a strongly coupled linear sigma model.

If the setting of small initial background oscillation is relevant in physical situation in high-energy hadronic collisions, a sharp peak in pion momentum distributions can be a clear signal for the parametric resonance mechanism, one of the candidate mechanism for DCC.

The potential possibility of large domain formation, the key issue in DCC, is not fully explored and hence unanswered in the present analysis. We hope that we can return to this problem by having a formalism without instability problem in the near future.

\section{Acknowledgment}

We thank Robert Brandenberger for bringing [7] to our attention. This work was supported in part by the Grant-in-Aid for Scientific Research No. 12640285, Ministry of Education, Culture, Sports, Science and Technology of Japan. The research of H.H. was partly supported by Kitasato University Research Grant for Young Researchers. 


\section{References}

[1] Early discussions on DCC may be found e.g., in: A. A. Anselm, Phys. Lett. B217 169 (1989); A. A. Anselm and M. G. Ryskin, Phys. Lett. B266 482 (1991); J.-P. Blaizot and A. Krzywicki, Phys. Rev. D46 246 (1992); Phys. Rev. D50 442 (1994); J. D. Bjorken, Acta Phys. Pol. B23 561 (1992); J. D. Bjorken, Acta Phys. Pol. B28 2773 (1997); J. D. Bjorken, K. L. Kowalski, and C. C. Taylor, Talk at 7th Rencontres de Physique de la Valee d'Aoste, La Thuile Rencontres 1993, page 507.

[2] Y. Fujimoto, C. M. G. Lattes, and S. Hasegawa, Phys. Rep. 65151 (1980); L. T. Baradzei et al., Nucl. Phys. B370 (1992) 365; J. Lord and J. Iwai (unpublished)

[3] K. Rajagopal and F. Wilczek, Nucl. Phys. B404 577 (1993); K. Rajagopal, Talk at 25th International Workshop on Gross Properties of Nuclei and Nuclear Excitation: QCD Phase Transitions, January 13-18, 1997 Hirschegg, Austria, hep-ph/9703258.

[4] M. Asakawa, Z. Huang, and X.-N. Wang, Phys. Rev. Lett. 743126 (1995).

[5] H. Hiro-Oka and H. Minakata, Phys. Rev. C61, 044903 (2000).

[6] H. Minakata, Nonequilibrium Aspects of DCC's, in Proceedings of RIKEN BNL Research Center Workshop on "Quantum Fields In $\&$ Out of Equilibrium", BNL-52560, pages 187-192, October 26-30, 1998.

[7] J. Traschen and R. Brandenberger, Phys. Rev. D42, 2491 (1990).

[8] L. Kofman, A. Linde, and A. A. Starobinsky, Phys. Rev. Lett. 73, 3195 (1994); Phys. Rev. D56, 3258 (1997).

[9] D. Boyanovsky, H. J. de Vega, and R. Holman, Phys. Rev. D51, 734 (1995); D. Boyanovsky, H. J. de Vega, R. Holman, D.-S. Lee, and A. Singh, ibid D51, 4419 (1995).

[10] M. Yoshimura, Prog. Theor. Phys. 94, 873 (1995); H. Fujisaki et al., Phys. Rev. D53, 6805 (1996); Phys. Rev. D54, 2494 (1996).

[11] S. Mrówczyński and B. Müller, Phys. Lett. B363, 1 (1995). 
[12] H. Hiro-Oka and H. Minakata, Phys. Lett B425, 129 (1998); Erratum ibid. B434, 461 (1998).

[13] H. P. Yuen, Phys. Rev. A13, 2226 (1976); D. F. Walls, Nature 306, 141 (1983); D. F. Smirnov and A. S. Troshin, Sov. Phys. Usp. 30, 851 (1987).

[14] D. I. Kaiser, Phys. Rev. D59, 117901 (1999).

[15] F. Cooper, Y. Kluger, E. Mottola, and J. E. Paz, Phys. Rev. D51, 2377 (1995); F. Cooper, S. Habib, Y. Kluger, and E. Mottola, ibid D55, 6471 (1997).

[16] D. Boyanovsky, H. J. de Vega, R. Holman, and S. P. Kumar, Phys. Rev. D56, 3929 (1997); ibid 5233 (1997).

[17] Y. Shtanov, J. Traschen and R. Brandenberger, Phys. Rev. D51, 5438 (1995).

[18] M. Abramowitz and I. A. Stegun, Handbook of Mathematical Functions (Dover Publication, New York, 1965). 


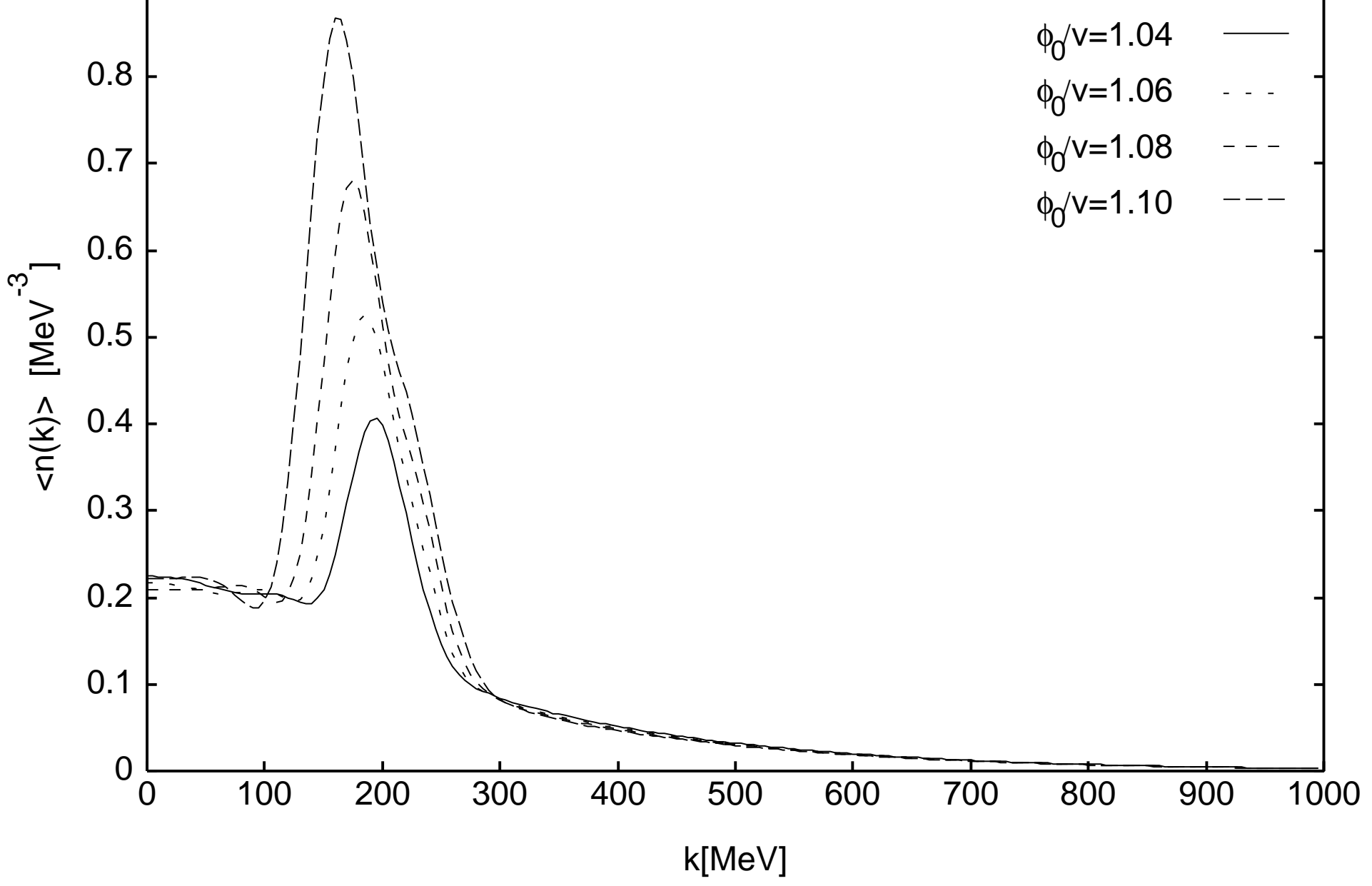




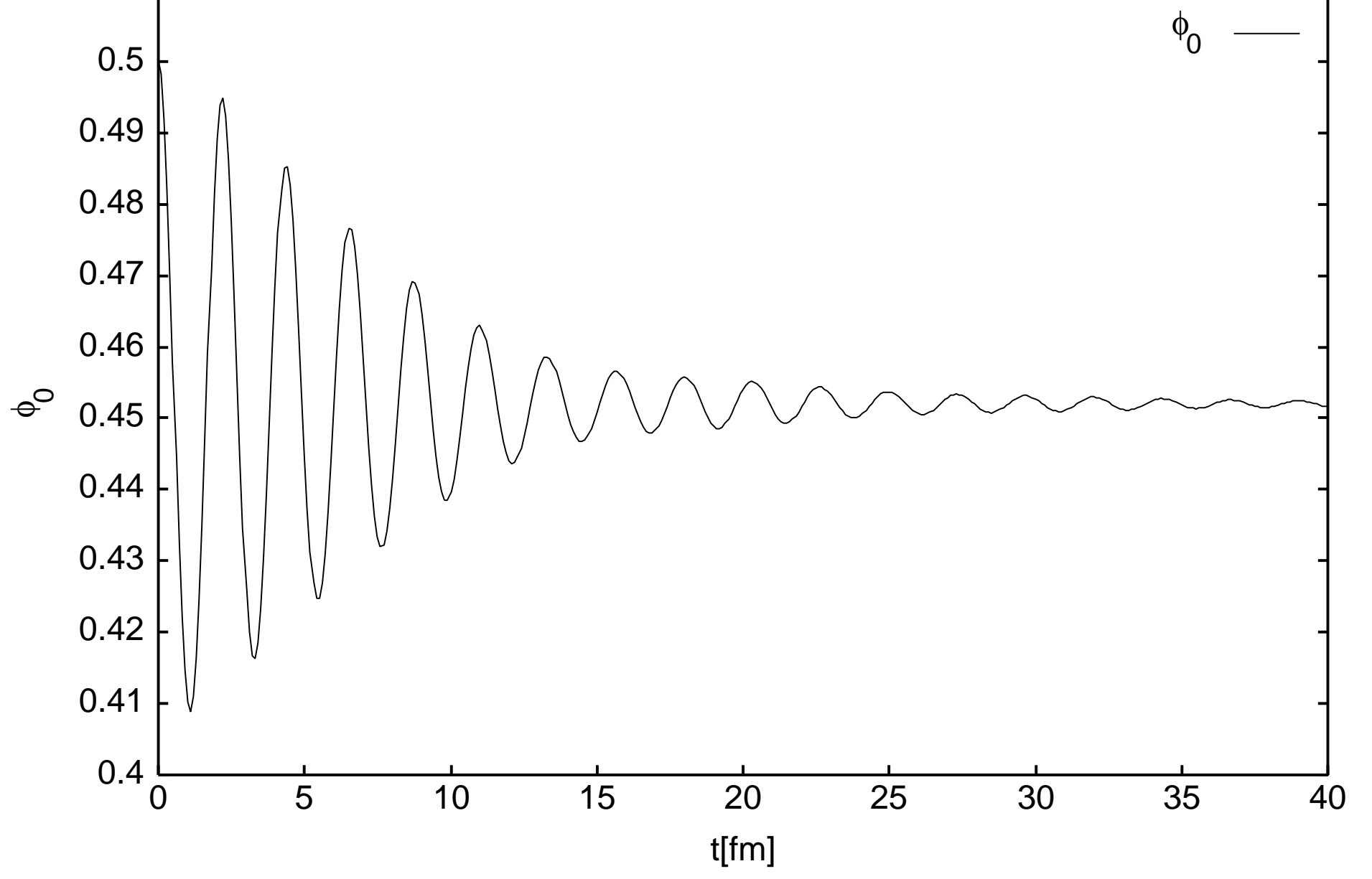


